\section{GPA auf einen Blick}

Die Gesellschaft für Pädiatrische Allergologie und Umweltmedizin e.V. (GPA) wurde vor zehn Jahren als Dachverband der vier bestehenden regionalen pädiatrisch-allergologischen Arbeitsgemeinschaften gegründet. Mittlerweile zählt die GPA fast 2.000 Mitglieder und ist damit die mitgliederstärkste Fachgesellschaft in der Deutschen Gesellschaft für Kinder- und Jugendmedizin e.V. (DGKJ). Zu den wesentlichen Aufgaben der GPA gehören:

— Förderung der Weiter- und Fortbildung allergologisch interessierter Kinderund Jugendärzte, beispielsweise durch die „Kompaktkurse Pädiatrische Allergologie", die einem von der GPA erstellten bundeseinheitlichen Curriculum folgen

— Beteiligung an der Qualitätssicherung im Bereich pädiatrische Allergologie

— Interessenvertretung der allergologisch tätigen Kinder- und Jugendärzte auf Bundesebene

— Zusammenarbeit mit anderen Organisationen im Bereich der Kinderheilkunde: Gesellschaft für pädiatrische Pneumologie e.V. (GPP), Deutsche Gesellschaft für Kinderheilkunde und Jugendmedizin e.V. (DGKJ), Berufsverband der Ärzte für Kinderheilkunde und Jugendmedizin Deutschlands e.V. (BVKJ), Deutsche Gesellschaft für Allergologie und klinische Immunologie e.V. (DGAKI), Ärzteverband Deutscher Allergologen e.V. (ÄDA), European Academy of Allergology and Clinical Immunology (EAACI) u. a.

— Herausgabe der Zeitschrift „Pädiatrische Allergologie in Klinik und Praxis"

_ Förderung der wissenschaftlichen Allergologie

— Veranstaltung von Symposien, Jahrestagungen und Workshops zu aktuellen Fragen aus dem Bereich der pädiatrischen Allergologie

— Vergabe des mit 5.000€ dotierten „Förderpreis Pädiatrische Allergologie“: Der von Pharmacia Diagnostics gestiftete Preis honoriert jährlich Veröffentlichungen oder Projekte von Einzelpersonen oder Gruppen, die in besonderer Weise zur Verbesserung der Situation von allergiekranken Kindern und Jugendlichen beigetragen haben. Über die Vergabe entscheidet ein Kuratorium aus wissenschaftlich tätigen Kinderallergologen sowie Vertretern der GPA und des Sponsors.
GPA News

\section{Fortbildung zum Thema Nahrungsmittelallergie}

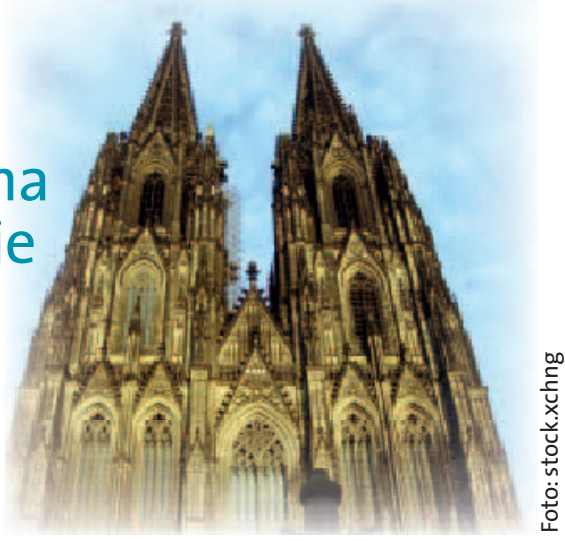

Für den Nahrungsmittelallergie-Tag der WAPPA in Köln konnten herausragende Experten gewonnen werden.

auch gegen Nahrungsmittelallergene. Nahrungsmittelallergien haben deshalb in den letzten Jahren Kollegen in Praxis und Klinik vor ganz neue Herausforderungen gestellt. Nicht nur Säuglinge mit Kuhmilch- und Hühnereiweißallergie erfordern hohe diagnostische und therapeutische Expertise, sondern zunehmend auch Kinder und Jugendliche mit Baumnuss- und Erdnussallergie. Hieraus ergeben sich ganz neue Fragestellungen: Muss man gegen Baumnuss oder Erdnuss Sensibilisierte provozieren? Wer braucht welches Notfallset? Müssen wir Anaphylaxie-Schulungen anbieten? Wie viel Sicherheit bieten uns die neuen Deklarationsvorschriften?

Einen vollständigen Überblick über die Themen gibt der Kasten links. Neben den Hauptvorträgen sollen die Workshops Gelegenheit geben, die Details in kleinen Gruppen zu diskutieren.

Ich freue mich auf ihre Teilnahme am Ersten Nahrungsmittelallergie-Tag der WAPPA!

Dr. Ernst Rietschel, Köln, Erster Vorsitzender der WAPPA

— Ernährungsberatung bei Baumnuss- und Erdnussallergie. U. Körner — Differenzialdiagnosen der Nahrungsmittelallergien. H. Skopnik

12.30-14.00 Mittagspause

14.00-15.00 Fälle. L. Lange (Moderation) 15.00-15.30 Pause

15.30-16.00 Deklaration und Nachweis von versteckten Allergenen in Nahrungsmitteln. S. Vieths

16.00-16.30 Pollenassoziierte Nahrungsmittelallergien. L. Lange

16.30-17.00 Therapie der Anaphylaxie durch Nahrungsmittelallergene. K. Beyer

17.00 Ende

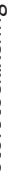

$\begin{array}{ll}\text { Programm } & \\ \text { Nahrungsmittel- } \\ \text { allergietag } \\ \text { der WAPPA }\end{array}$

Impressum „GPA-Nachrichten“

\section{Verantwortlich für den Inhalt}

Dr. Ernst Rietschel, Köln

\section{Redaktion \\ Markus Seidl}

Verlag

() Urban \& Vogel GmbH, München

\section{GPA-Geschäftsstelle}

Dr. Frank Friedrichs, Aachen

Tel.: (02 41) 9800486

Fax: (02 41) 9800259

E-Mail:info@gpaev.de 